\title{
Investigation of the WR 11 field at decimeter wavelengths ${ }^{\star}, \star \star$
}

\author{
P. Benagliaa ${ }^{1,2}$, S. del Palacio ${ }^{1,2}$, C. H. Ishwara-Chandra ${ }^{3}$, M. De Becker ${ }^{4}$, N. L. Isequilla ${ }^{2}$, and J. Saponara ${ }^{1,2}$ \\ ${ }^{1}$ Instituto Argentino de Radioastronomia, CONICET \& CICPBA, CC5 (1897) Villa Elisa, Provincia de Buenos Aires, Argentina \\ e-mail: paula@iar-conicet.gov.ar \\ 2 Facultad de Ciencias Astronómicas y Geofísicas, UNLP, Paseo del Bosque s/n, 1900, La Plata, Argentina \\ ${ }^{3}$ National Centre for Radio Astrophysics (NCRA-TIFR), Pune 411 007, India \\ ${ }^{4}$ Space sciences, Technologies and Astrophysics Research (STAR) Institute, University of Liège, Quartier Agora, 19c, \\ Allée du 6 Août, B5c, 4000 Sart Tilman, Belgium
}

Received 23 December 2018 / Accepted 21 March 2019

\begin{abstract}
The massive binary system WR 11 ( $\gamma^{2}$-Velorum) has recently been proposed as the counterpart of a Fermi source. If this association is correct, this system would be the second colliding wind binary detected in $\mathrm{GeV} \gamma$-rays. However, the reported flux measurements from 1.4 to $8.64 \mathrm{GHz}$ fail to establish the presence of nonthermal (synchrotron) emission from this source. Moreover, WR 11 is not the only radio source within the Fermi detection box. Other possible counterparts have been identified in archival data, some of which present strong nonthermal radio emission. We conducted arcsec-resolution observations toward WR 11 at very low frequencies (150$1400 \mathrm{MHz}$ ) where the nonthermal emission - if existent and not absorbed - is expected to dominate. We present a catalog of more than 400 radio emitters, among which a significant portion are detected at more than one frequency, including limited spectral index information. Twenty-one of these radio emitters are located within the Fermi significant emission. A search for counterparts for this last group pointed at MOST 0808-471; this source is $2^{\prime}$ away from WR 11 and is a promising candidate for high-energy emission, having a resolved structure along 325-1390 MHz. For this source, we reprocessed archive interferometric data up to $22.3 \mathrm{GHz}$ and obtained a nonthermal radio spectral index of $-0.97 \pm 0.09$. However, multiwavelength observations of this source are required to establish its nature and to assess whether it can produce (part of) the observed $\gamma$-rays. WR 11 spectrum follows a spectral index of $0.74 \pm 0.03$ from 150 to $230 \mathrm{GHz}$, consistent with thermal emission. We interpret that any putative synchrotron radiation from the colliding-wind region of this relatively short-period system is absorbed in the photospheres of the individual components. Notwithstanding, the new radio data allowed us to derive a mass-loss rate of $2.5 \times 10^{-5} M_{\odot} \mathrm{yr}^{-1}$, which, according to the latest models for $\gamma$-ray emission in WR 11 , would suffice to provide the required kinetic power to feed nonthermal radiation processes.
\end{abstract}

Key words. radio continuum: general - radio continuum: stars - radiation mechanisms: non-thermal - stars: individual: WR11

\section{Introduction}

Massive stars produce powerful stellar winds. In binary systems, these winds collide, constituting a colliding wind binary (CWB). In the wind collision region (WCR), the shocked winds heat up to several tens of MK. Colliding wind binaries are expected to radiate throughout the whole electromagnetic spectrum, from radio frequencies to $\gamma$-rays: the theoretical interest of CWBs as candidate $\gamma$-ray sources has been extensively studied in the literature (e.g., Eichler \& Usov 1993; White \& Chen 1995; Romero et al. 1999).

So far, more than 40 systems have been identified as relativistic particle accelerators (De Becker \& Raucq 2013), thanks to the evidence for nonthermal (NT) radiation found mainly in the radio domain. Such a radio emission is interpreted as synchrotron emission produced by a population of relativistic electrons. These relativistic particles are presumably accelerated in the shock fronts of the WCR by the diffusive shock acceleration (DSA) mechanism (Reimer et al. 2006; Pittard \& Dougherty

\footnotetext{
${ }^{\star}$ Full Table 4 is only available at the CDS via anonymous ftp to cdsarc.u-strasbg.fr (130.79.128.5) or via http://cdsarc. u-strasbg. fr/viz-bin/qcat?J/A+A/625/A99

$\star \star$ The radio data presented in this work were obtained with the Giant Metrewave Radio Telescope (GMRT). The GMRT is operated by the National Centre for Radio Astrophysics of the Tata Institute of Fundamental Research.
}

2006). In general, the radio emission from CWBs is potentially made of three contributions: one thermal, from the individual ionized stellar winds; another one thermal, from the shocked gas in the WCR; and one NT, from the relativistic electrons accelerated in the WCR. The radio emission from a single stellar wind is expected to be steady and to present a flux density $S_{v} \propto v^{\alpha}$, where $v$ is the frequency and the spectral index $\alpha$ is close to 0.6 (Wright \& Barlow 1975; Panagia \& Felli 1975). The NT radio emission from the WCR at low frequencies is notably higher than that expected for the thermal emission from individual stellar winds. Its spectral index is basically negative, and the flux density and spectral index may vary as a function of time. The thermal free-free emission from the WCR is also modulated with the orbital phase, both in flux density and spectral index. The latter can be close to 1 for the case of radiative shocks (Pittard 2010).

The only confirmed $\gamma$-ray emitting $\mathrm{CWB}$ is $\eta$-Car, as the high-energy $\gamma$-ray emission from this system presents a modulation corresponding to the orbital period of the binary (Tavani et al. 2009; Reitberger et al. 2015). Additionally, $\eta$-Car has been recently reported as a nonthermal hard X-ray source (Hamaguchi et al. 2018) and a very high-energy $\gamma$-ray source (Leser et al. 2017).

Pshirkov (2016) proposed WR 11 as the second CWB with a $\gamma$-ray counterpart after analyzing almost seven years of Pass- 8 Fermi data. He discovered a Fermi excess at a position coincident to this source with a flux of $(2.7 \pm 0.5) \times 10^{-12} \mathrm{erg} \mathrm{cm}^{-2} \mathrm{~s}^{-1}$. 
In general, the detection of $\gamma$-ray emission implies the presence of relativistic particles. If at least part of those particles are electrons, we could also expect to find the radio synchrotron emission these particles produce by interacting with ambient magnetic fields.

Archival radio emission from WR 11 presents a spectral index consistent with thermal bremsstrahlung. Nonetheless, this is not sufficient to rule out WR 11 as the counterpart of the Fermi source, as it is most likely that the synchrotron emission from the system - if existent - would be absorbed by the stellar winds material. If it were possible to detect $\gamma$-ray emission modulated with the period of WR 11, then it would be strong evidence supporting that this is the actual counterpart. However, the low high-energy flux does not allow for such timing analysis as the detected excess is found only after integrating during several years.

On the other hand, Benaglia (2016) studied the field of WR 11 by means of archive Australia Telescope Compact Array (ATCA) data and found several NT radio sources in the Fermi detection box which, in principle, could also be related to the counterpart of the Fermi source. In this work we carry out an investigation of the WR 11 field-of-view (FoV) sources below $1.4 \mathrm{GHz}$ to analyze which of these are clearly NT particle accelerators, and to infer if any of them could account for the Fermi excess. Section 2 summarizes what is known about WR 11 and its surroundings that is relevant to this study. In Sect. 3 we describe the observations and images generated from these observations. Section 4 presents the results obtained from the images and some analysis. A discussion mainly of the NT contributions to the radio emission is given in Sect. 5, and Sect. 6 closes with the conclusions.

\section{WR 11 and its surroundings}

Gamma ${ }^{2}$-Velorum or WR 11, (RA, $\quad \operatorname{Dec}_{\mathrm{J} 2000}=8: 9: 31.95$, $-47: 20: 11.71$ ), is the nearest stellar massive binary system, at a distance of $340 \mathrm{pc}$. It is composed by a WC8 and an O7.5 star. The orbit semimajor axis is $1.2 \mathrm{AU}$ and the system period is 79 days (North et al. 2007), and is thus prone to host a WCR. van der Hucht et al. (2007), analyzing XMM-Newton data, derived a mass-loss rate $\dot{M}=8 \times 10^{-5} M_{\odot} \mathrm{yr}^{-1}$ and detected X-rays dominated by thermal emission from the WCR.

A recent work by Pshirkov (2016) claimed that WR 11 is the counterpart of a Fermi $6.1 \sigma$ flux excess, which would make it the second CWB to be detected in $\mathrm{GeV}$ $\gamma$ rays. The author derived a flux in the high-energy range $(0.1-100 \mathrm{GeV})$ of $1.8 \pm 0.6 \times 10^{-9} \mathrm{ph} \mathrm{cm}^{-2} \mathrm{~s}^{-1}$ and an energy flux of $2.7 \pm 0.5 \times 10^{-12} \mathrm{erg} \mathrm{cm}^{-2} \mathrm{~s}^{-1}$. At a distance of $340 \mathrm{pc}$, the flux translates into a luminosity $L=3.7 \pm 0.7 \times 10^{31} \mathrm{erg} \mathrm{s}^{-1}$, which is well below the available wind kinetic luminosity. Numerical models by Reitberger et al. (2017) support the association of WR 11 with the Fermi source.

The NT nature of WR 11 radiation is still uncertain. This source was detected with ATCA at the lowest frequency of $1.4 \mathrm{GHz}$ with a flux of $9 \mathrm{mJy}$ (see Chapman et al. 1999, and references therein). The spectral indices derived from 1.4 to $8.64 \mathrm{GHz}$ resulted from 1.2 to 0.3 . However, Benaglia (2016) reduced archive ATCA data of three projects (identified as C599, C787, and C1616) and found very consistent results for WR 11. The emission spectral index between 1.4 and $2.4 \mathrm{GHz}$ is equal to $0.85 \pm 0.1$, regardless of the orbital phase. Multi-configuration C787 data averaged along 180 days (from June to December, 2001) allowed us to map the FoV of WR 11 and reveal many nearby NT sources. Besides WR 11, seven sources were detected above a nominal threshold of $10 \mathrm{mJy}$, all of which presented negative spectral indices. In particular, the so-called S6, a double source with intense NT radiation, is located at only $2^{\prime}$ from WR 11 inside the $6.1 \sigma$ probability contour of the Fermi excess.

We observed WR 11 and its surroundings with the Giant Metrewave Radio Telescope (GMRT) at decimeter wavelengths to investigate the emission regime of the CWB and to address whether other sources in the field could be responsible or contribute to the Fermi source.

\section{Radio observations and data reduction}

\subsection{GMRT data}

Dedicated observations pointing at WR 11 were carried out with the GMRT in four frequency bands, centered at 150, 325, 610, and $1390 \mathrm{MHz}$, using the back end nonpolarimetric configuration of $32 \mathrm{MHz}$ bandwidth; see Table 1 with the observing details (project code 30_033). The source had been observed with the ATCA at $1400 \mathrm{MHz}$ (Chapman et al. 1999) and we collected data at this band with the GMRT to compare both results. This allowed us to use data sets of the two radio interferometers in a common analysis. In addition, the GMRT angular resolution was $\sim 2-5$ times better than the ATCA angular resolution.

The source was observed during 2016 on 28 May (325 MHz, $6 \mathrm{~h}), 23 \mathrm{Jul}(610 \mathrm{MHz}, 4 \mathrm{~h})$, and 3 Oct (1390 MHz, $4 \mathrm{~h})$, and in 2017 on $21 \mathrm{Jan}$ and 11 Feb (150 MHz, $8 \mathrm{~h})$. The data reduction and imaging were performed with the Source Peeling and Atmospheric Modeling (SPAM) module (Intema 2014), a python-based extension to the Astronomical Imaging Processing System package (AIPS; Greisen 2003), for the 150, 325, and $610 \mathrm{MHz}$ band data sets. We used the Common Astronomy Software Applications (CASA; McMullin et al. 2007) to process the $1390 \mathrm{MHz}$ raw data in the standard way and obtained a robust-weighted self-calibrated image. The sources 3C147 and 3C286 were used as primary calibrators. The phase calibrators were $0828-375$ at $1390 \mathrm{MHz}$ and $0837-198$ for the rest of the bands. The images were built with robust weightings.

The MIRIAD software package (Sault et al. 1995) and the routine KVIS of the KARMA visualization software (Gooch 1996) were used for data analysis. The synthesized beams and the rms attained at the field center are given in Table 1.

\subsection{Archive data}

To complement the investigation, we made use of the Australia Telescope Online Archive (ATOA) ${ }^{1}$ observations that targeted WR 11, projects C787 and C1616. All data sets were processed with the MIRIAD package in a standard way, obtaining robustweighted images. The C787 reprocessed data consisted of $0.75 \mathrm{~h}$ on source at $4.8 \mathrm{GHz}$ and $3.79 \mathrm{~h}$ at $8.64 \mathrm{GHz}$ in $6 \mathrm{~A}$ array configuration (observations carried out in Dec 232001 and May 23 1999, respectively). The C1616-6A data sets comprised $4.5 \mathrm{~h}$ onsource time at $22.231 \mathrm{GHz}$, and $4.55 \mathrm{~h}$ at $22.367 \mathrm{GHz}$, imaged together.

\section{Results}

\subsection{WR 11 system}

WR 11 (source S5 of Benaglia 2016) was detected at 325, 610 , and $1390 \mathrm{MHz}$ GMRT images. The radio fluxes, obtained

\footnotetext{
1 https://atoa.atnf.csiro.au/
} 
Table 1. GMRT observing information and parameters of the images.

\begin{tabular}{lrrrr}
\hline \hline Frequency band center $(\mathrm{MHz})$ & 150 & 325 & 610 & 1390 \\
Phase calibrator & $0837-187$ & $0837-187$ & $0837-187$ & $0828-375$ \\
Observing date $(\mathrm{s})$ & $21 / \mathrm{Jan}, 11 / \mathrm{Feb} / 2017$ & $28 / \mathrm{May} / 2016$ & $23 / \mathrm{Ju} / / 2016$ & $03 / \mathrm{Oct} / 2016$ \\
Time on source $(\mathrm{min})$ & $186 \pm 6$ & 171 & 120 & 209 \\
Field of view $^{(a)}(\operatorname{arcmin})$ & $81 \pm 4$ & $43 \pm 3$ & $24 \pm 2$ \\
Synthesized beam $_{\text {Image center rms }\left(\mathrm{mJy} \mathrm{beam}^{-1}\right)}$ & $59.6^{\prime \prime} \times 14.0^{\prime \prime}, 2.5^{\circ}$ & $22.6^{\prime \prime} \times 6.4^{\prime \prime}, 8.2^{\circ}$ & $12.1^{\prime \prime} \times 3.2^{\prime \prime}, 12.5^{\circ}$ & $5.4^{\prime \prime} \times 2.1^{\prime \prime},-175.9^{\circ}$ \\
\hline
\end{tabular}

Notes. ${ }^{(a)}$ Taken from the GMRT Observer's Manual; www.ncra.tifr.res.in/ncra/gmrt/gmrt-users/observing-help/ manual_7jul15.pdf
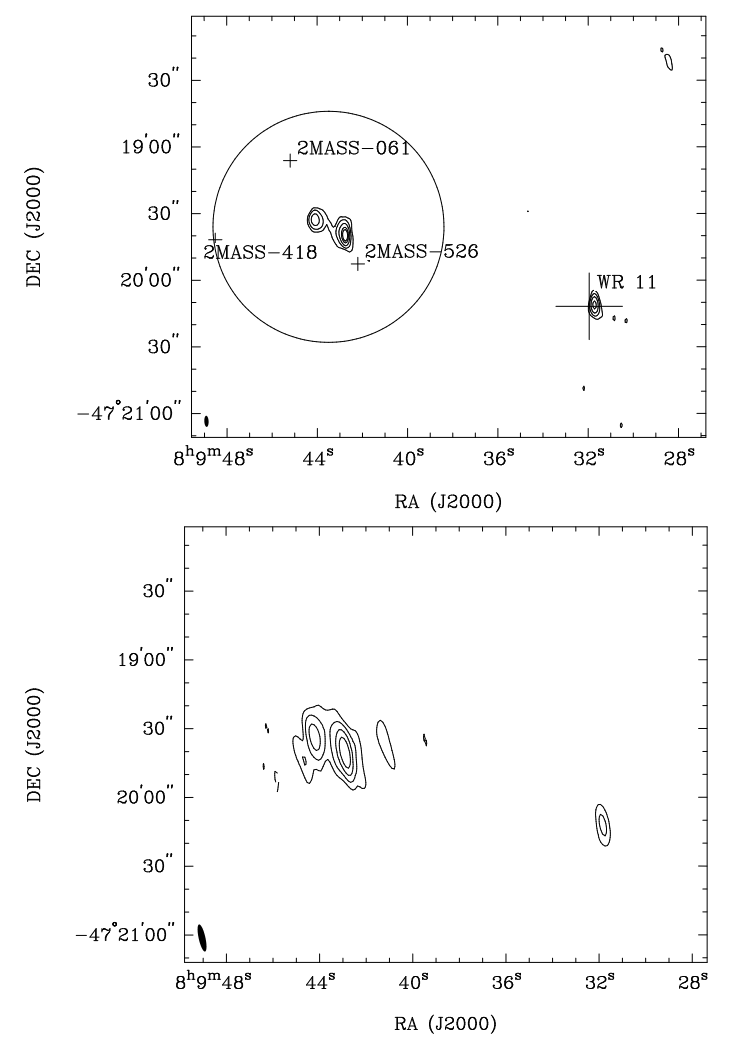

Fig. 1. Continuum images of WR 11 and MOST 0808-471. Top: at $1390 \mathrm{MHz}$. Contour levels are $\pm 3,10,30,80$, and $130 \sigma$, where $\sigma=0.07 \mathrm{mJy} \mathrm{beam}^{-1}$. The circle represents the beam of the Molonglo Observatory Synthesis Telescope (see text). The sources resulting from a Simbad database search are also denoted with crosses. 2MASS-061, 2MASS-418, and 2MASS-526 stand for 2MASS J08094519-4719061, 2MASS J08094851-4719418, and 2MASS J08094219-4719526, respectively. Bottom: at $610 \mathrm{MHz}$; contour levels of $\pm 3,10,30$, and $80 \sigma$, where $\sigma=0.16 \mathrm{mJy}^{-1}$ beam ${ }^{-1}$. Synthesized beams are shown at the bottom left corner of each image.

by means of a Gaussian fit, resulted in $2.9 \pm 0.3 \mathrm{mJy}$ at $325 \mathrm{MHz}, 3.1 \pm 0.2 \mathrm{mJy}$ at $610 \mathrm{MHz}$, and $10.5 \pm 1 \mathrm{mJy}$ at $1390 \mathrm{MHz}$. At $150 \mathrm{MHz}$, the rms around the position of WR 11 is $0.9 \mathrm{mJy} \mathrm{beam}^{-1}$, thus we quote a flux upper limit (UL) of 2.7 mJy. The images are shown in Figs. 1 and 2.

Table 2 lists radio fluxes of WR 11 derived in this work, together with others gathered from the literature, including the date of the observations. We checked the 1.4-8.64 GHz fluxes of Chapman et al. (1999) by reducing the ATCA archive data already mentioned and the $0.843 \mathrm{GHz}$ flux by measuring the
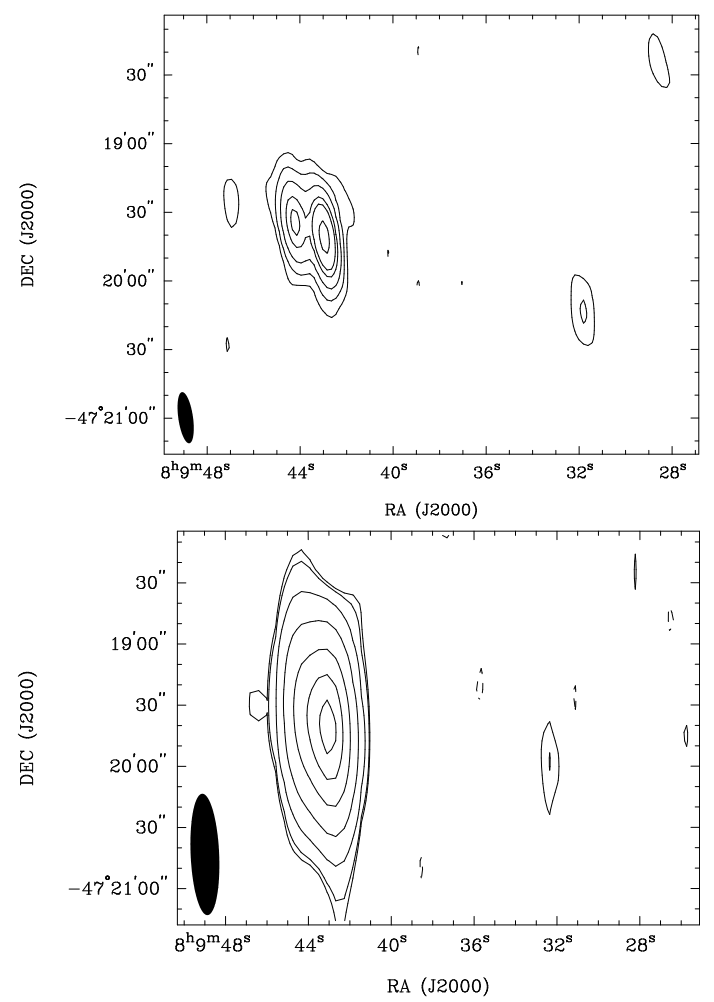

Fig. 2. Continuum images of WR 11 and MOST 0808-471. Top: at $325 \mathrm{MHz}$; contour levels of $\pm 3,10,30,80,130$, and $300 \sigma$, where $\sigma=0.2 \mathrm{mJy}$ beam $^{-1}$. The maxima, from right to left, correspond to sources I23 and I26 (double) of Table 5, respectively. Bottom: at $150 \mathrm{MHz}$; contour levels of $\pm 3,4,10,30,80,150$, and $220 \sigma$, where $\sigma=0.8 \mathrm{mJy}$ beam $^{-1}$. Synthesized beams are shown at the bottom left corner of each image.

value from the MGPS2 image, obtaining same values among error bars in all cases. In particular, we compared the $1.4 \mathrm{GHz}$ flux values taken using three different data sets (Chapman et al. 1999; Benaglia 2016, and this work). The consistency of the results supports the study of the spectral energy distribution (SED) of WR 11 from $150 \mathrm{MHz}$ to $\sim 22 \mathrm{GHz}$ combining GMRT and ATCA data. Moreover, we found that observations taken with similar array+configuration combinations at different years (1997 and 2001 for $2.4 \mathrm{GHz} ; 1995$ and 2001 for 4.8 and $8.64 \mathrm{GHz}$; 1997 and 2016 for $1.4 \mathrm{GHz}$ ) serve to derive flux values in very good agreement. We conclude that they form a uniform database, and also that they do not show flux variability at the mJy level. In addition, the WISE survey catalog (Cutri et al. 2012) gives the following fluxes of WR 11: $31.44 \pm 0.5 \mathrm{Jy}$ 
Table 2. Flux of the WR 11 system along the radio band.

\begin{tabular}{crccc}
\hline $\begin{array}{c}\text { Frequency } \\
(\mathrm{GHz})\end{array}$ & $\begin{array}{r}\text { Flux } \\
(\mathrm{mJy})\end{array}$ & $\begin{array}{c}\text { Synth. beam } \\
\left(\operatorname{arcsec}^{2}\right)\end{array}$ & $\begin{array}{c}\text { Obs. } \\
\text { date }\end{array}$ & Ref. \\
\hline 0.150 & $<2.7$ & $59.6 \times 14.0$ & 2017.09 & 1 \\
0.325 & $2.9 \pm 0.3$ & $22.6 \times 6.4$ & 2016.41 & 1 \\
0.610 & $3.1 \pm 0.2$ & $12.1 \times 3.2$ & 2016.56 & 1 \\
0.843 & $8.2 \pm 1$ & $43 \times 43$ & $1983-84$ & 2 \\
1.4 & $10 \pm 1$ & $6.3 \times 2.3$ & 2016.67 & 1 \\
1.4 & $9.23 \pm 0.13$ & $12 \times 8$ & 1997.15 & 3 \\
2.4 & $17.5 \pm 0.1$ & $8 \times 5$ & 1997.15 & 3 \\
4.8 & $26.5 \pm 0.28$ & $\sim 3 \times 3$ & 1995.49 & 4 \\
8.64 & $32.2 \pm 0.63$ & $\sim 1 \times 1$ & 1995.49 & 4 \\
14.7 & $67 \pm 10$ & $2.3 \times 2.3$ & 1977.47 & 5 \\
19.9 & $86 \pm 4$ & $10.8 \times 10.8$ & 2004.73 & 6 \\
22.3 & $96 \pm 3$ & $0.77 \times 0.36$ & 2006.92 & 1 \\
230 & $342 \pm 27$ & $25 \times 25$ & 1990.70 & 7 \\
\hline
\end{tabular}

References. (1) This work; (2) Jones (1985); (3) Chapman et al. (1999); (4) Leitherer et al. (1997); (5) Morton \& Wright (1978); (6) Murphy et al. (2009) (AT20G); (7) Leitherer \& Robert (1991).

at $3.4 \mu \mathrm{m}, 30.63 \pm 0.66 \mathrm{Jy}$ at $4.6 \mu \mathrm{m}, 15.77 \pm 0.15 \mathrm{Jy}$ at $12 \mu \mathrm{m}$, and $6.90 \pm 0.01 \mathrm{Jy}$ at $22 \mu \mathrm{m}$.

Figure 3 shows the SED of WR 11 from radio to infrared (IR) ranges. The SED is well fitted by a power law with spectral index $\alpha=0.74 \pm 0.03$, which is consistent with thermal free-free emission from the ionized stellar winds. A spectral index of 0.6 is the canonical value for individual stellar winds, but an index close to 0.7 might be explained in terms of the influence of acceleration and deceleration zones and/or changes of the ionization structure in the winds (e.g., Leitherer \& Robert 1991). We note that the WCR also produces thermal radio emission that might contribute to the observed fluxes ${ }^{2}$. An expected index of 1.1 was obtained by Pittard (2010) and Montes et al. (2011) through numerical calculations of CWBs when the WCR emission dominates, and Montes et al. (2009) observed radio emission with spectral indices $\sim 1$ in various WR systems. However, the WCR emission is expected to be modulated with the orbital phase (e.g., Pittard 2010); and the good fit shown in Fig. 3 for various observations at different epochs points to a rather steady emission. In addition, we should expect the likely thermal emission from the WCR to be significantly absorbed by the dense WC wind material, preventing its signature to be measured or even noticed. Therefore, we conclude that the observed SED from radio to IR in WR 11 is dominated by the individual winds and is not likely to be significantly contaminated with any emission (either thermal or NT) from the WCR structure.

\subsection{MOST 0808-471}

Using the Molonglo Observatory Synthesis Telescope (MOST), Jones (1985) detected a radio source at a distance of $2^{\prime}$ from WR 11 with a flux of $69 \pm 2 \mathrm{mJy}$, thereafter MOST 0808-471, brighter than WR 11 at $843 \mathrm{MHz}$. Thus, any measurement toward WR 11 performed with a beam larger than $2^{\prime}$ might be contaminated with emission from this source (at least at centimeter wavelengths). In Benaglia (2016) it was named as S6. Because of the close proximity of S6 to WR 11, and since one

2 The WCR can also produce NT radio emission with its negative spectral index; however, such emission is doomed to be absorbed in the stellar winds for such compact systems.

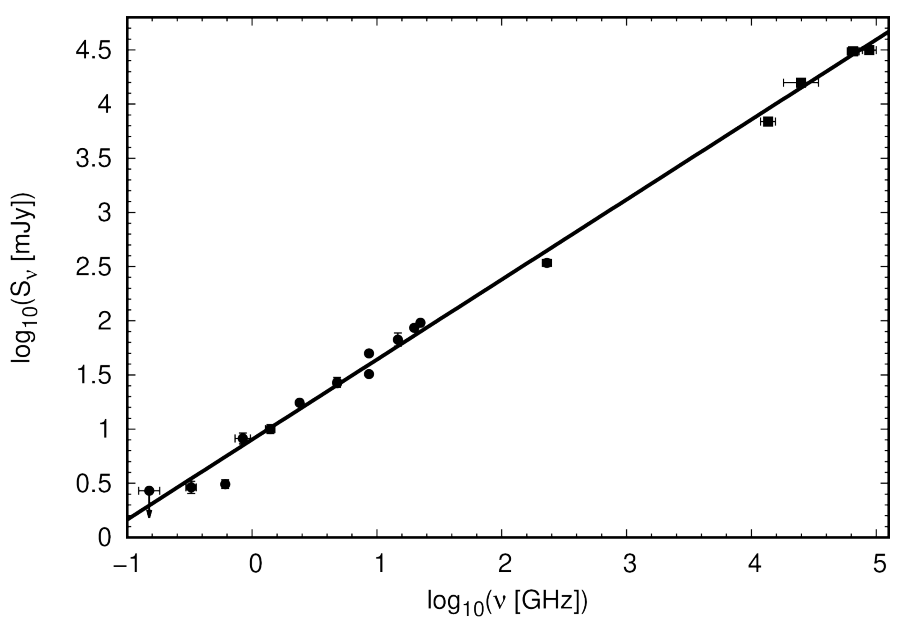

Fig. 3. Spectral energy distribution of WR 11, from radio (bullets) to IR (squares, WISE catalog; Cutri et al. 2012) frequencies. The spectral index value of the fit is $+0.74 \pm 0.03$.

Table 3. Radio fluxes of the source MOST 0808-471 up to $9 \mathrm{GHz}$.

\begin{tabular}{lrcc}
\hline \hline $\begin{array}{l}\text { Frequency } \\
(\mathrm{GHz})\end{array}$ & $\begin{array}{r}\text { Flux } \\
(\mathrm{mJy})\end{array}$ & Reference & Structure \\
\hline 0.150 & $330 \pm 10$ & This work & Unresolved \\
0.325 & $140 \pm 1$ & This work & Resolved \\
$\quad$ Left lobe & $44.6 \pm 0.22$ & & \\
$\quad$ Right lobe & $96.3 \pm 0.23$ & & \\
0.610 & $66 \pm 3$ & This work & Resolved \\
Left lobe & $17.3 \pm 0.3$ & & \\
$\quad$ Right lobe & $50.4 \pm 0.2$ & & \\
0.843 & $69 \pm 2$ & 1 & Unresolved \\
1.39 & $39 \pm 3$ & This work & Resolved \\
Left lobe & $8.7 \pm 0.6$ & & \\
Right lobe & $24.6 \pm 1.6$ & & \\
2.4 & $25 \pm 2$ & 2 & Unresolved \\
4.8 & $6.5 \pm 1$ & This work & Unresolved \\
8.64 & $2.0 \pm 0.3$ & This work & Unresolved \\
\hline
\end{tabular}

References. (1) Jones (1985); (2) Benaglia (2016).

of the goals of the present investigation is to reveal whether other source(s) besides WR 11 could be responsible or contribute to the Fermi excess detected by Pshirkov (2016), we provide a more detailed description of the results for this source.

The images of MOST 0808-471 at 150, 325, 610, and $1390 \mathrm{MHz}$ are presented in Figs. 1 and 2. The fluxes of MOST 0808-471 at 150, 325, and 610 were obtained performing Gaussian fitting to the GMRT images. The value at $1390 \mathrm{MHz}$, the frequency at which the source was resolved, was estimated measuring the flux above $3 \sigma$. The S6 flux as estimated from the MGPS2 survey image cutouts (Murphy et al. 2007) agrees with that of Jones (1985). The 4.8 and $8.64 \mathrm{GHz}$ flux values were derived from the images produced using the archive data mentioned in Sect. 3.

The series of measurements presented in Table 3 allowed us to build the very first radio SED of MOST 0808-471, shown in Fig. 4. The plot reveals a clear nonthermal radio spectrum over about two decades of frequencies. A discussion of the nature of this spectrum is presented in Sect. 5.2. 


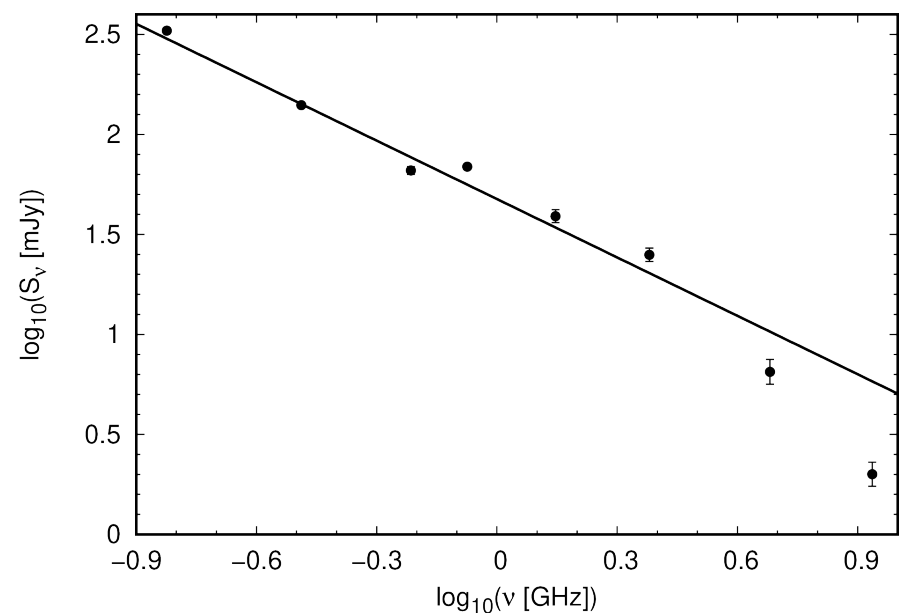

Fig. 4. Radio flux of MOST 0808-471 as a function of the observing frequency. The spectral index value of the fit is $-0.97 \pm 0.09$.

\subsection{Other sources in the field}

Benaglia (2016) carried out a first search for sources at the 24 arcmin diameter ATCA field toward WR 11, listing seven additional sources, including MOST 0808-471. The GMRT images at 150, 325, and $610 \mathrm{MHz}$ allow a deeper and more extended quest for counterparts. The FoVs corresponding to those bands are given in Table 1 .

We searched for radio continuum sources within each image using the Python Blob Detector and Source Finder (PyBDSF) ${ }^{3}$. This tool decomposes a radio interferometric image into bidimensional Gaussians and provides a list of sources and their parameters. In the process, the background rms is computed. When variations along the FoV are statistically significant, which is the case in our images, an rms map is built by estimating the rms value in few-pixels-size boxes; the detection threshold thus increases toward image edges. We defined the threshold to separate source and noise pixels as $7 \mathrm{rms}^{4}$. For checking the scope of the results, individual monitoring of random sources yielded no fake detections. Source sizes were not limited.

The PyBDSF run resulted in 410 sources at $150 \mathrm{MHz}$ (tagged G150-1 to -410), 224 at $325 \mathrm{MHz}$ (tagged G325-1 to -224), and 66 at $610 \mathrm{MHz}$ (tagged G610-1 to -66). In Table 4 we list the parameters of the first sources found at each band: ID (Col. 1), coordinates, and integrated flux of the fitted Gaussian functions (Cols. 2-4), the peak flux (Col. 5), the coordinates of the emission maximum (Cols. 6 and 7), and the major and minor axes and position angle of the fitted function (Cols. 8-10). The full list is available at the CDS. We also present in Fig. 5 histograms of the number of sources as a function of their flux at 150 and $325 \mathrm{MHz}$.

As a second step, we focused on the sources lying within a circular region of $43^{\prime}$ in diameter, which corresponds to the smallest FoV of the three lower observing bands $(610 \mathrm{MHz})$. The data sets produced by the PyBDSF tool allowed us to perform spectral index estimations. In this region we found $53325-\mathrm{MHz}$ sources with emission at $150 \mathrm{MHz}$ and/or at $610 \mathrm{MHz}$ above $7 \sigma$ level. The sources are named as I01 to I53. The coordinates (Cols. 2 and 3), fluxes (Cols. 4-6), spectral indices $\alpha$ (Cols. 7 and 8 ), and sizes (fitted beam major and minor axes and position

\footnotetext{
3 http://www . astron.nl/citt/pybdsf/

4 With these settings, the number of sources found on average were $\sim 98 \%$ of those found for $5 \mathrm{rms}$.
}
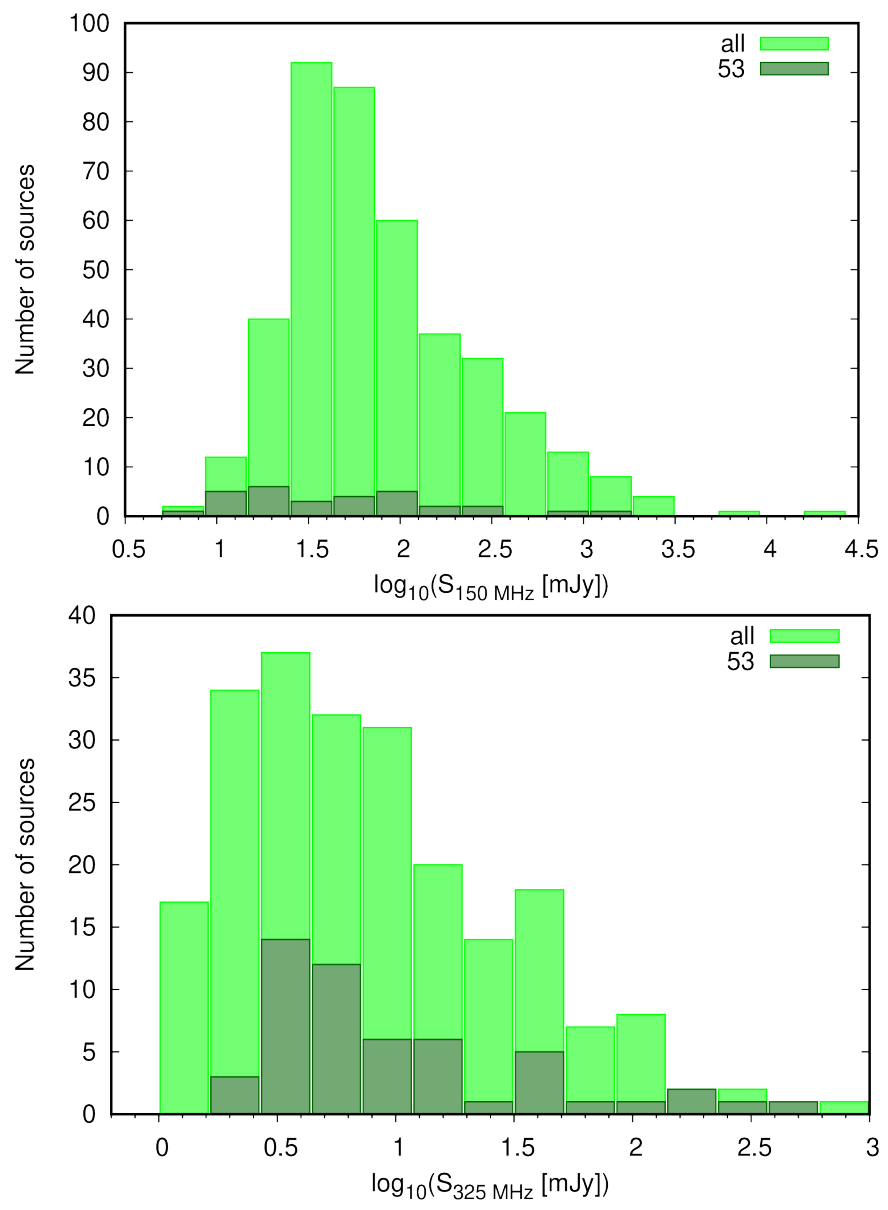

Fig. 5. Histograms of fluxes. Top: sources at $150 \mathrm{MHz}$. Bottom: sources at $325 \mathrm{MHz}$. Light green boxes indicate the whole sample of sources detected at each wavelength, whereas in dark green the sample is restricted to the 53 sources described in Sect. 4.3.

angle, Col. 9) are listed in Table 5, and are shown separately in the histograms of Fig. 5. A distinction must be made regarding the spectral index values derived for these 53 sources. In the case in which the source fills the beam at both frequency bands, the spectral index corresponds to an average along the source regardless of its structure. The $\alpha$ values quoted for unresolved sources have to be taken with caution, since the pair of images to derive the spectral index were not corrected to the same beam. Such a detailed analysis is presented elsewhere. In Fig. 6 we show histograms of the spectral indices obtained for these sources. In Table 6 we list the cross-identifications among the sources found at more than one band (ID number, from I01 to I53) and the names given at the three different GMRT observed bands (G150-, G325-, and G610-).

We carried out a Simbad search for counterparts over the region covered by the extension of the fitted source of Table 5 at the highest frequency (either $610 \mathrm{MHz}$ when it was detected at that band, or $325 \mathrm{MHz}$ for the same reason). Positive results are listed in Table 7. There we quote the source ID (the same as in Table 5), the distance to a Simbad possible counterpart, the name and type of this latter, and whether the source lies within the Fermi excess. In this way, in this last table we indicate which sources with derived spectral indices were spatially coincident with the Fermi excess reported by Pshirkov (2016); there are 21 of such sources over the area portrayed by Pshirkov (2016) and Benaglia (2016). 
Table 4. Sources detected at 150, 325, and $610 \mathrm{MHz}$ above the $7 \sigma$ level (first records).

\begin{tabular}{|c|c|c|c|c|c|c|c|c|c|}
\hline ID & $\begin{array}{r}\mathrm{RA}_{\mathrm{IJ2000}} \\
(\mathrm{hms})\end{array}$ & $\begin{array}{r}\operatorname{Dec}_{\mathrm{J} 2000} \\
(\mathrm{dms})\end{array}$ & $\begin{array}{r}\text { Total flux } \\
\text { (mJy) }\end{array}$ & $\begin{array}{r}\text { Peak flux } \\
\left(\mathrm{mJy}_{\text {beam }}^{-1}\right)\end{array}$ & $\begin{array}{r}\mathrm{RA}_{\max } \\
(\mathrm{hms})\end{array}$ & $\begin{array}{r}\operatorname{Dec}_{\max } \\
(\mathrm{dms})\end{array}$ & $\begin{array}{r}B_{\text {maj }} \\
(")\end{array}$ & $\begin{array}{r}B_{\min } \\
\left({ }^{\prime \prime}\right)\end{array}$ & $\begin{array}{l}B_{\mathrm{pa}} \\
\left(^{\circ}\right)\end{array}$ \\
\hline \multicolumn{10}{|c|}{ Detected at $150 \mathrm{MHz}$} \\
\hline$\overline{\mathrm{G} 150-1}$ & $8: 21: 03.5 \pm 0.12$ & $-47: 36: 05 \pm 6.4$ & $125.1 \pm 8.6$ & $61.8 \pm 5.0$ & $8: 21: 03.5 \pm 0.12$ & $-47: 36: 05 \pm 6.4$ & $100.5 \pm 15.6$ & $16.9 \pm 0.7$ & $12.7 \pm 1.4$ \\
\hline G150-2 & $8: 20: 46.1 \pm 0.01$ & $-47: 36: 07 \pm 0.1$ & $622.6 \pm 33.5$ & $4302.5 \pm 6.8$ & $8: 20: 46.5 \pm 0.01$ & $-47: 36: 11 \pm 0.1$ & $78.8 \pm 0.2$ & $41.7 \pm 0.1$ & $161.3 \pm 0.2$ \\
\hline G150-3 & $8: 20: 39.8 \pm 0.05$ & $-47: 34: 39 \pm 3.5$ & $307.0 \pm 7.8$ & $87.8 \pm 6.0$ & $8: 20: 39.8 \pm 0.05$ & $-47: 34: 39 \pm 3.5$ & $3.1 \pm 8.3$ & $31.4 \pm 1.7$ & $175.1 \pm 2.8$ \\
\hline G150-4 & $8: 20: 41.4 \pm 0.12$ & $-47: 38: 52 \pm 6.3$ & $36.6 \pm 11.0$ & $34.0 \pm 5.4$ & $8: 20: 41.4 \pm 0.12$ & $-47: 38: 52 \pm 6.3$ & $54.4 \pm 15.3$ & $16.5 \pm 1.5$ & $12.8 \pm 5.8$ \\
\hline G150-5 & $8: 20: 45.2 \pm 0.03$ & $-47: 38: 49 \pm 4.3$ & $14.6 \pm 14.6$ & $33.8 \pm 3.6$ & $8: 20: 45.2 \pm 0.03$ & $-47: 38: 49 \pm 4.3$ & $37.7 \pm 10.2$ & $9.6 \pm 0.4$ & $3.4 \pm 1.6$ \\
\hline G150-6 & $8: 20: 27.7 \pm 0.03$ & $-46: 55: 30 \pm 2.0$ & $89.8 \pm 5.4$ & $59.7 \pm 3.2$ & $8: 20: 27.7 \pm 0.03$ & $-46: 55: 30 \pm 2.0$ & $8.5 \pm 4.7$ & $21.5 \pm 0.8$ & $4.5 \pm 1.4$ \\
\hline G150-7 & $8: 20: 33.1 \pm 0.02$ & $-47: 35: 48 \pm 0.1$ & $60.0 \pm 11.8$ & $691.6 \pm 6.2$ & $8: 20: 34.7 \pm 0.02$ & $-47: 35: 47 \pm 0.1$ & $7.4 \pm 0.7$ & $28.6 \pm 0.2$ & $175.1 \pm 0.8$ \\
\hline G150-8 & $8: 20: 13.1 \pm 0.02$ & $-46: 46: 45 \pm 1.6$ & $119.4 \pm 4.9$ & $70.6 \pm 3.1$ & $8: 20: 13.1 \pm 0.02$ & $-46: 46: 45 \pm 1.6$ & $59.0 \pm 3.7$ & $24.0 \pm 0.8$ & $0.3 \pm 1.1$ \\
\hline G150-9 & $8: 20: 19.3 \pm 0.02$ & $-47: 42: 52 \pm 1.5$ & $439.6 \pm 7.1$ & $172.3 \pm 5.1$ & $8: 20: 19.3 \pm 0.02$ & $-47: 42: 52 \pm 1.5$ & $82.5 \pm 3.4$ & $25.9 \pm 0.5$ & $173.5 \pm 179.9$ \\
\hline G150-10 & $8: 20: 05.2 \pm 0.05$ & $-46: 54: 59 \pm 4.4$ & $47.5 \pm 4.9$ & $27.8 \pm 3.1$ & $8: 20: 05.2 \pm 0.05$ & $-46: 54: 59 \pm 4.4$ & $63.5 \pm 10.5$ & $22.5 \pm 1.7$ & $0.2 \pm 5.0$ \\
\hline \multicolumn{10}{|c|}{ Detected at $325 \mathrm{MHz}$} \\
\hline G325-1 & $8: 14: 45.5 \pm 0.01$ & $-47: 21: 41 \pm 0.1$ & $63.9 \pm 0.5$ & $44.1 \pm 0.3$ & $8: 14: 45.5 \pm 0.01$ & $-47: 21: 41 \pm 0.1$ & $3.5 \pm 0.2$ & $8.9 \pm 0.0$ & $6.1 \pm 179.4$ \\
\hline G325-2 & $8: 14: 37.4 \pm 0.02$ & $-47: 02: 29 \pm 1.2$ & \pm 0.4 & \pm 0.3 & $8: 14: 37.4 \pm 0.02$ & $-47: 02: 29 \pm 1.2$ & $7.1 \pm 3.0$ & $11.2 \pm 0.7$ & $4.5 \pm 5.0$ \\
\hline G325-3 & $8: 14: 30.3 \pm 0.01$ & $-47: 29: 26 \pm 0.1$ & $52.1 \pm 0.5$ & $35.3 \pm 0.3$ & $8: 14: 30.3 \pm 0.01$ & $-47: 29: 26 \pm 0.1$ & $23.8 \pm 0.3$ & $9.0 \pm 0.1$ & $6.4 \pm 179.6$ \\
\hline G325-4 & $8: 14: 23.6 \pm 0.01$ & $-47: 38: 09 \pm 0.7$ & $10.9 \pm 0.5$ & $6.7 \pm 0.3$ & $8: 14: 23.6 \pm 0.01$ & $-47: 38: 09 \pm 0.7$ & $5.6 \pm 1.7$ & $9.2 \pm 0.3$ & $4.4 \pm 1.9$ \\
\hline G325-5 & $8: 13: 45.4 \pm 0.01$ & $-46: 47: 22 \pm 0.7$ & $8.6 \pm 0.5$ & $6.4 \pm 0.3$ & $8: 13: 45.4 \pm 0.01$ & $-46: 47: 22 \pm 0.7$ & $24.8 \pm 1.6$ & $7.9 \pm 0.2$ & $7.4 \pm 1.4$ \\
\hline G325-6 & $8: 13: 45.7 \pm 0.01$ & $-47: 14: 27 \pm 0.1$ & $77.8 \pm 0.4$ & $56.9 \pm 0.2$ & $8: 13: 45.7 \pm 0.01$ & $-47: 14: 27 \pm 0.1$ & $3.7 \pm 0.1$ & $8.4 \pm 0.0$ & $6.8 \pm 179.4$ \\
\hline G325-7 & $8: 13: 43.0 \pm 0.01$ & $-47: 47: 30 \pm 1.2$ & $4.7 \pm 0.4$ & $3.6 \pm 0.2$ & $8: 13: 43.0 \pm 0.01$ & $-47: 47: 30 \pm 1.2$ & $25.2 \pm 2.8$ & $7.5 \pm 0.3$ & $5.1 \pm 2.7$ \\
\hline G325-8 & $8: 13: 40.3 \pm 0.01$ & $-47: 55: 37 \pm 0.1$ & $126.6 \pm 0.6$ & $76.1 \pm 0.3$ & $8: 13: 40.5 \pm 0.01$ & $-47: 55: 36 \pm 0.1$ & $24.4 \pm 0.1$ & $9.7 \pm 0.0$ & $1.8 \pm 0.3$ \\
\hline G325-9 & $8: 13: 32.3 \pm 0.01$ & $-47: 31: 09 \pm 0.2$ & $20.2 \pm 0.3$ & $15.3 \pm 0.2$ & $8: 13: 32.3 \pm 0.01$ & $-47: 31: 09 \pm 0.2$ & $23.8 \pm 0.5$ & $8.1 \pm 0.1$ & $6.6 \pm 180.0$ \\
\hline G325-10 & $8: 13: 32.7 \pm 0.01$ & $-47: 40: 31 \pm 0.7$ & $6.3 \pm 0.4$ & $4.8 \pm 0.2$ & $8: 13: 32.7 \pm 0.01$ & $-47: 40: 31 \pm 0.7$ & $24.2 \pm 1.7$ & $8.0 \pm 0.2$ & $7.2 \pm 1.7$ \\
\hline \multicolumn{10}{|c|}{ Detected at $610 \mathrm{MHz}$} \\
\hline G610-1 & $8: 11: 46.0 \pm 0.01$ & $-47: 26: 13 \pm 0.8$ & $2.2 \pm 0.2$ & $1.4 \pm 0.1$ & $8: 11: 46.0 \pm 0.01$ & $-47: 26: 13 \pm 0.8$ & $2.7 \pm 1.9$ & $4.8 \pm 0.3$ & $7.4 \pm 6.1$ \\
\hline G610-2 & $8: 11: 39.8 \pm 0.02$ & $-47: 15: 42 \pm 1.0$ & $1.4 \pm 0.2$ & $1.1 \pm 0.1$ & $8: 11: 39.8 \pm 0.02$ & $-47: 15: 42 \pm 1.0$ & $13.6 \pm 2.3$ & $3.5 \pm 0.2$ & $14.4 \pm 3.8$ \\
\hline G610-3 & $8: 11: 17.2 \pm 0.01$ & $-47: 25: 40 \pm 0.3$ & $3.7 \pm 0.2$ & $2.9 \pm 0.1$ & $8: 11: 17.2 \pm 0.01$ & $-47: 25: 40 \pm 0.3$ & $12.5 \pm 0.6$ & $3.9 \pm 0.1$ & $10.7 \pm 1.3$ \\
\hline G610-4 & $8: 11: 16.6 \pm 0.01$ & $-47: 10: 55 \pm 0.9$ & $0.9 \pm 0.2$ & $0.9 \pm 0.1$ & $8: 11: 16.6 \pm 0.01$ & $-47: 10: 55 \pm 0.9$ & $11.8 \pm 2.1$ & $3.4 \pm 0.2$ & $12.1 \pm 4.4$ \\
\hline G610-5 & $8: 11: 09.6 \pm 0.01$ & $-46: 57: 49 \pm 0.6$ & $2.2 \pm 0.3$ & $2.3 \pm 0.1$ & $8: 11: 09.6 \pm 0.01$ & $-46: 57: 49 \pm 0.6$ & $12.8 \pm 1.5$ & $3.0 \pm 0.1$ & $7.9 \pm 2.0$ \\
\hline G610-6 & $8: 11: 03.8 \pm 0.01$ & $-47: 20: 09 \pm 0.2$ & $3.5 \pm 0.1$ & $3.2 \pm 0.1$ & $8: 11: 03.8 \pm 0.01$ & $-47: 20: 09 \pm 0.2$ & $12.3 \pm 0.5$ & $3.4 \pm 0.0$ & $10.6 \pm 0.8$ \\
\hline G610-7 & $8: 10: 55.9 \pm 0.01$ & $-47: 20: 13 \pm 0.5$ & $1.3 \pm 0.1$ & $1.2 \pm 0.1$ & $8: 10: 55.9 \pm 0.01$ & $-47: 20: 13 \pm 0.5$ & $12.5 \pm 1.3$ & $3.3 \pm 0.1$ & $12.5 \pm 2.2$ \\
\hline G610-8 & $8: 10: 55.7 \pm 0.01$ & $-47: 28: 54 \pm 0.5$ & $2.8 \pm 0.1$ & $1.6 \pm 0.1$ & $8: 10: 55.7 \pm 0.01$ & $-47: 28: 54 \pm 0.5$ & $14.1 \pm 1.1$ & $4.6 \pm 0.2$ & $13.5 \pm 2.8$ \\
\hline G610-9 & $8: 10: 55.0 \pm 0.01$ & $-47: 28: 58 \pm 0.2$ & & $3.7 \pm 0.1$ & $8: 10: 55.0 \pm 0.01$ & $-47: 28: 58 \pm 0.2$ & $13.3 \pm 0.4$ & $6.9 \pm 0.2$ & $8.3 \pm 2.2$ \\
\hline G610-10 & $8: 10: 31.2 \pm 0.01$ & $-47: 28: 22 \pm 0.5$ & $1.3 \pm 0.1$ & $1.3 \pm 0.1$ & $8: 10: 31.2 \pm 0.01$ & $-47: 28: 22 \pm 0.5$ & $12.0 \pm 1.1$ & $3.2 \pm 0.1$ & $9.9 \pm 1.9$ \\
\hline
\end{tabular}

Notes. $B_{\mathrm{maj}}, B_{\mathrm{min}}$ are the beam major and minor axes, and $B_{\mathrm{pa}}$ the beam position angle of the Gaussian fit by the pyBDSM routines. The full table is available at the CDS.

\section{Discussion}

\subsection{WR 11 system}

According to Reitberger et al. (2017), the reported $\gamma$-ray emission by Pshirkov (2016) can only be achieved by WR 11 in a hadronic model, as the predicted leptonic $\gamma$-ray emission is far too weak. The authors show that the $\gamma$-ray spectrum can be fitted with a $\mathrm{p}-\mathrm{p}$ component for certain combinations of an energy-dependent diffusion coefficient in the WCR, a proton injection ratio $\eta_{\mathrm{p}} \sim 10^{-3}$, and considering a mass-loss rate of the WR star of $\dot{M}_{\mathrm{WR}}=3 \times 10^{-5} M_{\odot} \mathrm{yr}^{-1}$. However, the authors note that a lower value of $\dot{M}_{\mathrm{WR}}=8 \times 10^{-6} M_{\odot} \mathrm{yr}^{-1}$ leads to an unrealistically high energetics requirement. It is thus relevant to explore deeper the issue of the actual mass-loss rate.

Our setl of measurements consistently point to a thermal origin for the radio emission from WR 11 from 0.3 to $230 \mathrm{GHz}$, refuting previous suggestions of the presence of a NT component at low frequencies (Chapman et al. 1999). The SED shown in Fig. 3 does not present any hint for a NT emission component contributing to the measured radio flux, which can thus be interpreted as optically thick thermal emission from stellar winds as described, for instance, by Wright \& Barlow (1975). First of all, it should be kept in mind that the WR 11 system is made up of two stars, both of which are likely to contribute to the thermal free-free emission. Let us estimate the potential contribution of the O-star to the measured flux densities, using the relations published by Wright \& Barlow (1975). According to Lamberts et al. (2017), the mass of the O-star companion should be about 28 solar masses. For an O7.5 spectral type, according to the calibration of O-type star parameters published by Martins et al. (2005), this points to a giant luminosity class. We note, however, that Lamberts et al. (2017) suggested that the best-matching IR spectrum is that of an O6.5I star, but such a spectral classification would be at odds with the mass of about 28 solar masses which should be very robust. In addition, let us caution that the O-type spectrum analyzed by Lamberts et al. (2017) is very likely contaminated by some emission from the CWR. This contamination casts some doubt on any luminosity class determination based only on the IR spectrum. We therefore assume the O7.5III classification for the $\mathrm{O}$ star. Adopting the mass-loss rate and terminal velocity proposed by Muijres et al. (2012) for that spectral classification, we obtain a flux density lower than $0.1 \mathrm{mJy}$ at $1.4 \mathrm{GHz}$. In addition, we note that the free-free emission from the O-star wind should be significantly reduced considering that a significant part of that wind is smashed by the collision with the WR 
P. Benaglia et al.: Investigation of the WR11 field at decimeter wavelengths

Table 5. Sources found above $7 \sigma$ level at more than one frequency band, inside the 610-MHz FoV.

\begin{tabular}{|c|c|c|c|c|c|c|c|c|}
\hline ID & $\begin{array}{r}\mathrm{RA}_{\mathrm{J} 2000} \\
(\mathrm{hms})\end{array}$ & $\begin{array}{r}\mathrm{Dec}_{\mathrm{J} 2000} \\
(\mathrm{dms})\end{array}$ & $\begin{array}{r}S_{150 \mathrm{MHz}} \\
(\mathrm{mJy})\end{array}$ & $\begin{array}{r}S_{325 \mathrm{MHz}} \\
(\mathrm{mJy})\end{array}$ & $\begin{array}{r}S_{610 \mathrm{MHz}} \\
(\mathrm{mJy})\end{array}$ & $\alpha_{150 \mathrm{MHz}}^{325 \mathrm{MHz}}$ & $\alpha_{325 \mathrm{MHz}}^{610 \mathrm{MHz}}$ & $\begin{array}{r}B_{\mathrm{maj}}, B_{\min }, B_{\mathrm{pa}} \\
\left({ }^{\prime \prime},{ }^{\prime \prime},{ }^{\circ}\right)\end{array}$ \\
\hline I01 & & & & & & .3 & -0 & $22.3,7.0,11.3$ \\
\hline 2 & $7.8 \pm$ & & $6.2 \pm$ & & & $1.1 \pm 0.03$ & & $12.5,9.5$ \\
\hline I03 & $: 07: 26.2 \pm 0.01$ & $47: 21: 26 \pm 0.2$ & & & $.5 \pm 0.28$ & $.8 \pm 0.28$ & $1.2 \pm 0.20$ & $23.7,7.1,7.5$ \\
\hline 04 & & & & & & & & \\
\hline I05 & $7+0+0$ & & $<7.8$ & & $2 \pm 0.20$ & $>-0.8$ & $-1.1 \pm 0.41$ & $24.6,6.8,7.8$ \\
\hline I06 & $1.7 \pm 0$ & $3: 49 \pm$ & $83.1 \pm 1.9$ & & & $-1.2 \pm 0.07$ & $-1.7 \pm 0.07$ & $23.2,7.1,8.6$ \\
\hline I07 & $1.9 \pm 0.01$ & $-47: 37: 06 \pm 1.2$ & & & & $-2.6 \pm 0.13$ & $-1.3 \pm 0.38$ & $37.3,7.6,6.6$ \\
\hline I08 & \pm 0.01 & & & 4 & 9 & & & $.9,176.2$ \\
\hline I09 & $9 \pm 0.01$ & .1 & & & & .03 & & $2.9,147.0$ \\
\hline I10 & $.7 \pm 0$ & & & & & $1.3 \pm 0.01$ & & $, 7.2,10.8$ \\
\hline I11 & 1 & 7 & & & & $>-0.9$ & $-0.5 \pm$ & $.5,6.9,9.1$ \\
\hline $\mathrm{I} 12$ & & & & & & & & \\
\hline I13 & 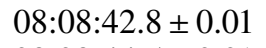 & & & & & & & \\
\hline I14 & & & & & & & & 7.7 \\
\hline I15 & & & & & & & & $, 12.7,5.4$ \\
\hline I16 & $0+0,0$ & 1 & & & & 05 & & , 6.6, 8.2 \\
\hline $\mathrm{I} 17$ & $8 \pm 0.01$ & 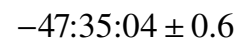 & & & & & 9 & , 7.1, 8.3 \\
\hline $\mathrm{I} 18$ & & & & & & & & $5.4,10.7$ \\
\hline I19 & & & & & & & & \\
\hline $\mathrm{I} 20$ & 1 & & & & & .51 & & $6.6,9.1$ \\
\hline I21 & $7+0 \Omega 1$ & 4 & & & & $>-1.2$ & & $1,6.9,7.8$ \\
\hline $\mathrm{I} 22$ & & & & & & & & 7.2 \\
\hline $\mathrm{I} 23$ & & & & & & & & \\
\hline $\mathrm{I} 24$ & & & & & & & & 7.2 \\
\hline $\mathrm{I} 25$ & 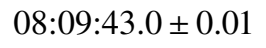 & & & & 5 & 26 & & 8.1 \\
\hline $\mathrm{I} 26$ & 2 & 1 & 2.7 & & & 02 & & 0,10 \\
\hline $\mathrm{I} 27$ & & & & & & & & 8.0 \\
\hline I28 & & & & & & & & 19.5 \\
\hline I 29 & 1 & -47 & .1 & & & -1 & &, 5.8 \\
\hline $\mathrm{I} 30$ & & & & & & & & 8.5 \\
\hline I31 & & & & & & & &, 3.3 \\
\hline I32 & & & & & & & & 10.0 \\
\hline I33 & $6 \pm 0.01$ & 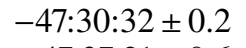 & & & & & & 4.5 \\
\hline I34 & $0 \pm 0.01$ & & & & & $>-0.4$ & & , 6.1 \\
\hline $\mathrm{I} 35$ & & & & & & $0 \pm 0.29$ & & 5.7 \\
\hline I36 & & & & & & 6 & & 7.8 \\
\hline I37 & & & & & & & & $2.8,7.4,5.9$ \\
\hline $\mathrm{I} 38$ & $3 \pm 0.01$ & 47 & & & & $>-0.8$ & & $23.1,7.8,6.7$ \\
\hline I39 & & 17 & & & & & & $23.1,7.2,8.3$ \\
\hline $\mathrm{I} 40$ & & & $3 \pm$ & & 2 & 7 & & 0.5 \\
\hline I41 & c & 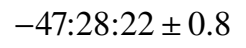 & $<7.5$ & & $1.4 \pm($ & & -1.0 & 6.1 \\
\hline $\mathrm{I} 42$ & & & & & & & & $24.5,11.8,9.0$ \\
\hline $\mathrm{I} 43$ & $7 \pm 0.01$ & & & & & $-1.4 \pm 0.59$ & $<-2.2$ & $26.9,7.7,6.9$ \\
\hline $\mathrm{I} 44$ & & 17. & & & & & & $4.9,12.3,12.9$ \\
\hline $\mathrm{I} 45$ & & & & & & & & \\
\hline $\mathrm{I} 46$ & $8 \pm 0$ & -47 & & & $3 \pm 0.17$ & & $-0.6 \pm$ & $22.9,6.8,7.78$ \\
\hline I47 & $9.6 \pm 0.01$ & $-46: 57: 48 \pm 0.2$ & \pm 1.8 & $13.0 \pm 0$ & $.4 \pm 0.34$ & $1.4 \pm 0.15$ & $-2.7 \pm$ & $23.2,6.5,9.0$ \\
\hline $\mathrm{I} 48$ & $66+001$ & $17,10.55,06$ & & & & & $-2.1 \pm$ & $6.8,8.3$ \\
\hline I49 & & -47 & $<7.4$ & & \pm 0 & $>-0.4$ & & $24.1,7.5,4.1$ \\
\hline $\mathrm{I} 50$ & $.8 \pm 0.01$ & $-47: 1$ & $<7.1$ & & $1.5 \pm 0.22$ & $>-1.6$ & $-0.6 \pm$ & $20.9,6.5,7.3$ \\
\hline I51 & $6.1 \pm 0.01$ & $-47: 26: 14 \pm 0$ & $<7.4$ & $4.1 \pm 0$ & $2.4 \pm 0.24$ & $>-0.8$ & $-0.9 \pm 0.43$ & $24.4,7.0,6.8$ \\
\hline I52 & 08 & $-47: 24: 54 \pm$ & & & & $-1.0 \pm 0.24$ & $<-3.2$ & $32.2,12.4,9.7$ \\
\hline I53 & $08: 12: 17.0 \pm 0.01$ & $-47: 23: 36 \pm 0.4$ & $48.5 \pm 1.8$ & $14.8 \pm 0.22$ & $<1.4$ & $-1.5 \pm 0.12$ & $<-3.7$ & $29.0,11.6,17.8$ \\
\hline
\end{tabular}

Notes. Flux ULs were calculated as $7 \times \mathrm{rms}$ at the source position. $B_{\mathrm{maj}}, B_{\min }$ are the beam major and minor axes at $325 \mathrm{MHz}$, and $B_{\mathrm{pa}}$ the beam position angle of the Gaussian fit by the pyBDSM routines. The source tagged as I26 corresponds to the position of MOST 0808-471 and is resolved in two and three components at 325 and $610 \mathrm{MHz}$, respectively; see Table 6 and also Sect. 4.2. 

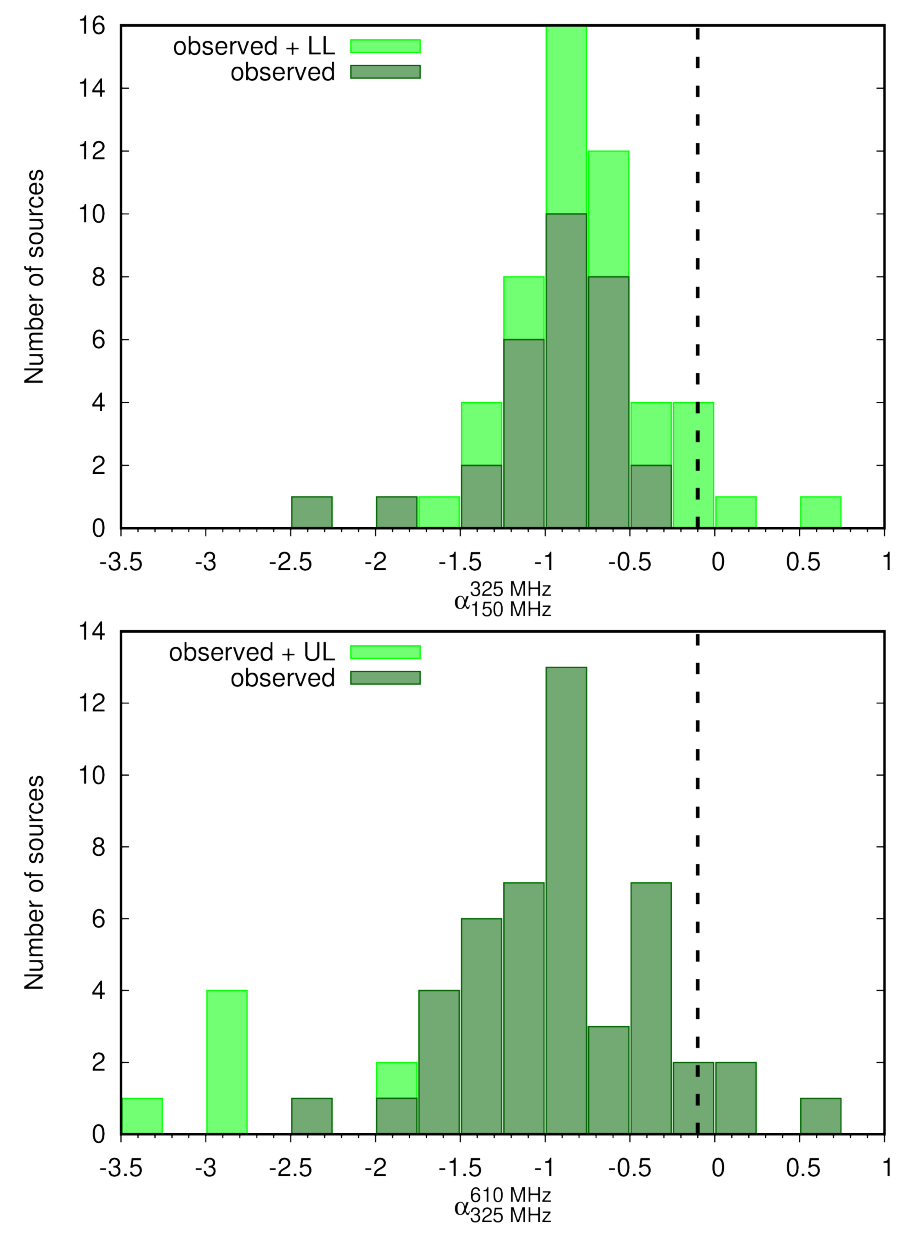

Fig. 6. Histograms of spectral indices using the values reported in Table 5. The dashed vertical line denotes $\alpha=-0.1$, which is the lowest possible value for thermal emission. Top: between fluxes at 150 and $325 \mathrm{MHz}$; when only an UL to the flux at $150 \mathrm{MHz}$ was available, we also estimated a lower limit (LL) to the spectral index. Bottom: between fluxes at 325 and $610 \mathrm{MHz}$; when only an UL to the flux at $610 \mathrm{MHz}$ was available, we also estimated an UL to the spectral index.

wind. This is supposed to lead to a further reduction of the freefree emission from that wind with respect to our estimate. Noting that the measured flux density at $1.4 \mathrm{GHz}$ is $10 \mathrm{mJy}$, we can conclude that the radio measurements are clearly dominated by the free-free emission from the WC8 wind.

We thus estimated the mass-loss rate of the WC wind using the following equation (adapted from Wright \& Barlow 1975):

$\dot{M}=4 \pi \mu m_{\mathrm{H}} v_{\infty}\left[\frac{2}{\pi K_{v} \gamma}\right]^{1 / 2}\left[\frac{S_{v}}{f_{\mathrm{cl}}^{2 / 3}} \frac{d^{2}}{1.342 \pi B_{v}}\right]^{3 / 4}$.

The expression for $K_{v}$ can be found in Wright \& Barlow (1975), and $B_{v}$ stands for Planck's function, which can be considered in the Rayleigh-Jeans limit at radio frequencies. Assuming a WC wind made only of $\mathrm{He}$, we adopted a mean molecular weight $(\mu)$ value of 4.0 , along with an rms ionic charge $(\gamma)$ of 1.0 (for details, see Leitherer et al. 1995). The clumping factor $\left(f_{\mathrm{cl}}\right)$ was assumed to be equal to 4.0, which agrees with the predictions for outer parts of massive star winds made by Runacres \& Owocki (2002), where the measured free-free radio emission is coming from $^{5}$. The parameter $T$ is the electron temperature set to be $50 \%$

\footnotetext{
5 As discussed notably by Puls et al. (2008), mass-loss rates corrected for the effect of wind clumping are reduced by a factor $\sqrt{f_{\mathrm{cl}}}$. As the flux
}

of the effective temperature (Drew 1990). The latter temperature for a WC8 star is expected to be about $70000 \mathrm{~K}$ (see Crowther 2007). We adopted a terminal velocity $\left(v_{\infty}\right)$ of $2000 \mathrm{~km} \mathrm{~s}^{-1}$. Following this approach, we obtained a mass-loss rate for the WC star on the order of $2.4 \times 10^{-5} M_{\odot} \mathrm{yr}^{-1}$. This value is very close to the value adopted by Reitberger et al. (2017) for their simulations (see above in this section). Such a value seems thus high enough to provide the required kinetic power to significantly feed NT emission processes.

In the framework of the potential contribution of WR 11 to the $\gamma$-ray emission reported on by Pshirkov (2016), the lack on NT radio emission deserves some comments. In very short period binary systems, free-free absorption (FFA) is expected to severely reduce any putative synchrotron radio emission produced in the WCR. In order to achieve a view of the capability of the stellar winds in the system to absorb significantly radio photons, we calculated the radius of the so-called radio photosphere for a FFA radial optical depth equal to one in a wide range of photon wavelengths. This calculation is performed using equations given by Wright \& Barlow (1975), adopting the same approach as De Becker et al. (2019) for the massive binary WR 133. The curves were computed for both stellar winds adopting the parameters specified above in this section, and are shown in Fig. 7. First, it is clear that FFA in the system is dominated by the WC wind. Second, the extension of the high optical depth zone is much larger than the typical dimension of the full binary system. According to Lamberts et al. (2017), the semimajor axis of the orbit is about 3.5 milli-arsec, which translates to a linear semimajor axis of about $250 R_{\odot}$ at a distance of about $340 \mathrm{pc}$ (in agreement with the results published by North et al. 2007). This is much smaller than the expected size of the radio photosphere at our selected wavelength, which is much larger than a few $1000 R_{\odot}$ at all frequencies below $300 \mathrm{GHz}$ (Fig. 7). The putative synchrotron emitting region in the system is thus completely and deeply embedded in the high FFA opacity region. Any synchrotron emission originating from the WCR would therefore be completely absorbed by the opaque WC wind. As a result, the lack of evidence for NT emission in the radio domain cannot be interpreted as evidence for the absence of any NT process at work in the system. This issue has already been discussed by De Becker et al. (2017), as for instance in the case of the CWB $\eta$-Car both NT X-rays and $\gamma$-rays have been detected but not NT radio emission.

\subsection{MOST $0808-471$}

The SED presented in Fig. 4 was first fitted by a power law, taking into account all the flux densities available for this source. The derived spectral index is $\alpha=-0.97 \pm 0.09$, which is consistent with NT synchrotron emission produced by a population of relativistic electrons with a power-law energy distribution with index $p=1-2 \alpha \approx-2.9$ (for a distribution defined as $N(E) \propto$ $E^{p}$, where $E$ is the electron energy). Such a steep synchrotron radio spectrum may potentially be explained by a population of relativistic electrons accelerated by shocks (through the DSA mechanism) characterized by a compression ratio lower than the expected value for strong, adiabatic shocks. In the latter case, the compression ratio is expected to be $\chi=4$. The linear DSA process predicts thus an electron index $p=-\frac{\chi+2}{\chi-1}=-2$.

density is proportional to the mass-loss rate to the $4 / 3$ power (Wright \& Barlow 1975), this translates into a correction of flux densities by a factor $f_{\mathrm{cl}}^{2 / 3}$. For $f_{\mathrm{cl}}=4$, we achieve the expected reduction of the mass-loss rate by a factor $\sqrt{2}$. 
P. Benaglia et al.: Investigation of the WR11 field at decimeter wavelengths

Table 6. Cross identification of sources detected at more than one band.

\begin{tabular}{lrrr|rrrr|rrrr}
\hline \hline ID & G325- & G150- & G610- & ID & G325- & G150- & G610- & ID & G325- & G150- & G610- \\
\hline I01 & 190 & - & 66 & I19 & 140 & 213 & 37 & I37 & 98 & - & 16 \\
I02 & 181 & 252 & 65 & I20 & 139 & 210 & 36 & I38 & 89 & - & 14 \\
I03 & 179 & 250 & 64 & I21 & 134 & - & 35 & I39 & 88 & - & 13 \\
I04 & 178 & 248 & - & I22 & 128 & - & 34 & I40 & 84 & 169 & 11,12 \\
I05 & 173 & - & 63 & I23 & 126 & - & 33 & I41 & 81 & - & 10 \\
I06 & 171 & 240 & 62 & I24 & 125 & 199 & 32 & I42 & 78 & 166 & - \\
I07 & 169 & 239 & 61 & I25 & 120 & 195 & 30 & I43 & 72 & 162 & -19 \\
I08 & 167 & 236 & $53-60$ & I26 & 114,115 & 192 & $26-28$ & I44 & 71 & 161 & 8,9 \\
I09 & 161 & 229 & $48-50$ & I27 & 119 & - & 29 & I45 & 70 & - & 7 \\
I10 & 162 & 230 & $47,51,52$ & I28 & 111 & 185 & 24,25 & I46 & 67 & - & 6 \\
I11 & 154 & - & 45 & I29 & 110 & 186 & 23 & I47 & 64 & 157 & 5 \\
I12 & 155 & - & 46 & I30 & 109 & 184 & 22 & I48 & 61 & - & 4 \\
I13 & 153 & - & 44 & I31 & 108 & 182 & 21 & I49 & 60 & - & 3 \\
I14 & 152 & - & 43 & I32 & 106 & 183 & - & I50 & 47 & - & 2 \\
I15 & 151 & 221 & 41,42 & I33 & 104 & 181 & 20 & I51 & 46 & - & 1 \\
I16 & 150 & 220 & 40 & I34 & 103 & - & 19 & I52 & 36 & 148 & - \\
I17 & 148 & - & 39 & I35 & 102 & 180 & 18 & I53 & 33 & 137 & - \\
I18 & 142 & - & 38 & I36 & 99 & 179 & 17 & & & & \\
\hline
\end{tabular}

Notes. ID is the same as in Table 5; G325- (G150-, G610-) is the name/number of the source detected at $325 \mathrm{MHz}(150 \mathrm{MHz}, 610 \mathrm{MHz})$ as in full Table 4, provided as online material.

Table 7. Literature-searched sources at the position of GMRT sources with spectral index information.

\begin{tabular}{lrlll}
\hline \hline ID & Dist. & Nearest source name & Nearest source type & Comments \\
\hline I03 & $9.1^{\prime \prime}$ & 2MASS J08072621-4721354 & YSO candidate & \\
I08 & $2.8^{\prime \prime}$ & 2MASS J08080880-4732335 & YSO candidate & \\
& $13^{\prime \prime}$ & 2MASS J08080942-4732249 & YSO candidate & \\
I09 & $18.8^{\prime \prime}$ & 2MASS J08082235-4710594 & YSO & Fermi excess \\
I23 & $1.9^{\prime \prime}$ & WR 11 & WR star & Fermi excess \\
I26 & $7.3^{\prime \prime}$ & MOST 0808-471 & Radio source & Fermi excess \\
& $14.1^{\prime \prime}$ & 2MASS J08094219-4719526 & Pre-main-sequence star & \\
I36 & $7.2^{\prime \prime}$ & IRAS 08084-4656 & IR point source & Fermi excess \\
I49 & $10.3^{\prime \prime}$ & 2MASS J08111607-4711036 & YSO candidate & \\
\hline
\end{tabular}

Notes. ID: same as in Table 5; Dist: distance between the Simbad source and the detected source at the $325 \mathrm{MHz}$ image; "Fermi excess" means that the $325 \mathrm{MHz}$ source lays within the Fermi excess presented by Pshirkov (2016).

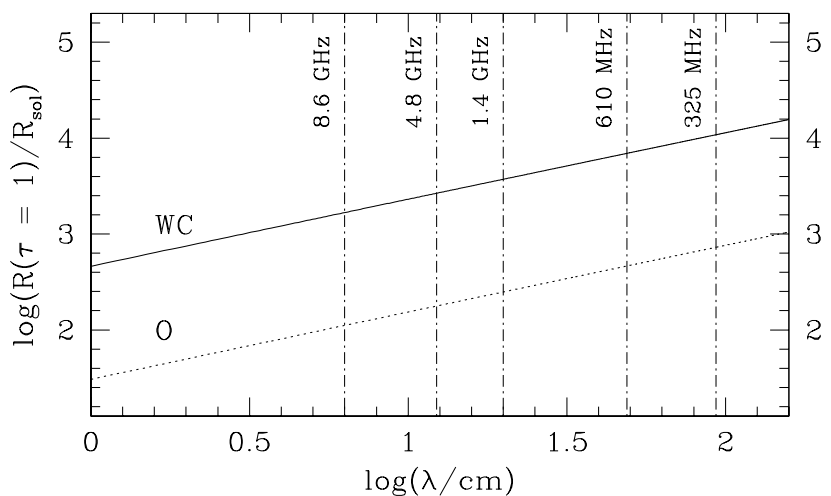

Fig. 7. Radio photosphere of the stellar winds in WR 11 as a function of radio wavelength. Vertical dashed lines emphasize some characteristic frequencies specified in the plot. The thick lines and the dotted lines represent the photosphere sizes of the $\mathrm{WC}$ and $\mathrm{O}$ components, respectively.
However, any compression ratio lower than 4 leads to an electron index lower than -2 , and consequently a steeper synchrotron spectrum. Such a situation happens for instance in young supernova remnants, where the high efficiency of particle acceleration leads to a significant feedback of the particle acceleration on the shock properties. This results in a deceleration of the upstream flow close to the shock discontinuity, leading to a reduction of the velocity jump, and accordingly to a reduction of the effective compression ratio (see, e.g., Reynolds et al. 2012, for a discussion of supernova remnants). This effect - referred to as shock modification - may thus in principle explain a significant deviation with respect to the canonical index resulting from DSA-generated relativistic electron populations. If this interpretation applies to MOST 0808-471, this would indicate a quite high particle acceleration efficiency. Otherwise, shock modification would not be significant.

However, the data plotted in the SED presented in Fig. 4 suggest a slightly flatter spectrum below $4.8 \mathrm{GHz}$, followed by a steeper spectrum at higher frequencies. The determination of 


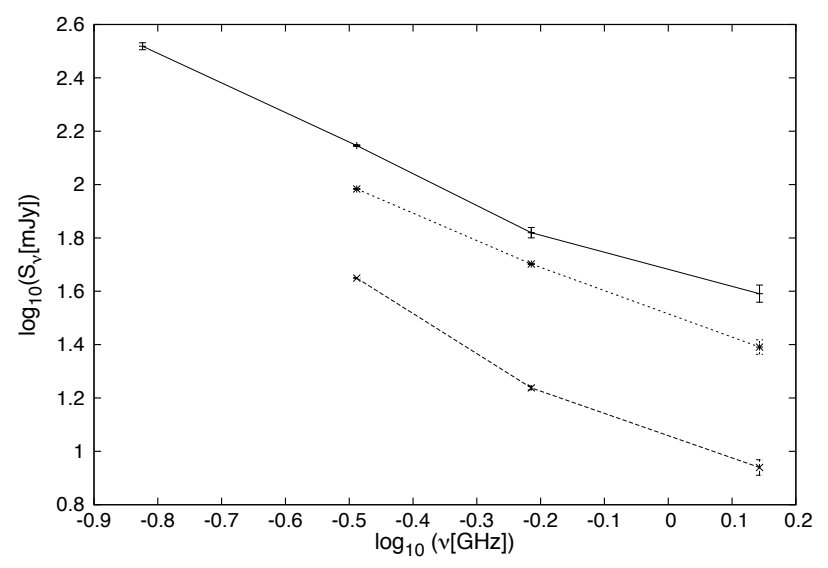

Fig. 8. GMRT fluxes of the MOST 0808-471 counterpart components. Dashed line: left component; dotted line: right component; solid line: total flux.

the spectral index below $4.8 \mathrm{GHz}$ yields $\alpha=-0.91 \pm 0.06$ (translating into $p \approx-2.8$ ), which is still quite steep. The unusual values of the spectral index, along with the apparent steepening at higher radio frequencies may also point to an inhomogeneous distribution of the magnetic energy density across the emitting region. It is known that the typical synchrotron photon frequency is proportional to the magnetic field strength and to the square of the emitting electron energy (see, e.g., Rybicki \& Lightman 1979). For a given population of relativistic electrons distributed across the source, different parts of the emitting region characterized by different magnetic field strengths may affect the measured 5 synchrotron emission that is averaged over the full emitting region. A larger emitting volume with a lower magnetic field leads to a stronger contribution at lower frequencies, whilst a smaller volume with a stronger magnetic field drives the emission from a smaller amount of relativistic electrons at higher frequencies, thus less contributing to the spectrum.

Alternatively, we should also consider the gradual cooling of relativistic electrons, where the more energetic electrons radiate at a higher rate than less energetic electrons. This may lead to a gradual steepening of effective electron distribution whose signature is measured through the synchrotron spectrum. Depending on the source properties, the cooling may be only due to synchrotron radiation, or may also be influenced by inverse Compton scattering in the Thomson regime. However, in the absence of clear indication on the nature of MOST 0808-471, we can only speculate as to the interpretation of the measured radio spectrum.

The images at the position of MOST 0808-471 obtained from the GMRT observations presented in this work are the most sensitive with the highest angular resolution at radio-centimeter frequencies. In Figs. 1 and 2 GMRT counterparts can be identified. At $v \geq 325 \mathrm{MHz}$, MOST 0808-471 appears resolved in more than one component, all of which, in principle, may or may not be related to the same physical object: a double-lobe single object or a set of two line-of-sight objects are possible. In Table 3 we discriminate the fluxes computed for left and right counterparts (named left and right lobes for simplicity); see also Fig. 8. The spectral indices are $\bar{\alpha} \approx-1.2$ and $\bar{\alpha} \approx-0.9$ for the left and the right lobes. Provided the two lobes are physically related, a straightforward hypothesis is that MOST 0808-471 consists of a NT bipolar jet source. Such an object is expected in various well-known scenarios. For instance, Rodríguez-Kamenetzky et al. (2016) described the synchrotron emission from young stellar object (YSO) jets (see also the A99, page 10 of 11 review by Anglada et al. 2018), and complemented the study with theoretical models that predict that these objects can produce $\gamma$-ray emission as well, involving bipolar jets (e.g., Araudo et al. 2007). Microquasars were also widely studied as $\gamma$-ray emitters with two-lobed radio counterparts (Romero et al. 2003). If the two lobes related to MOST 0808-471 belong to a single object at a distance of $360 \mathrm{pc}$, their projected separation is $\sim 5000 \mathrm{AU}$. Another possibility is that we are dealing with an extragalactic source such as a quasar. These objects are well known to be NT emitters from radio to $\gamma$ rays (see, for instance, Tadhunter 2016; Ackermann et al. 2015; Rieger 2017).

At the present stage, the information compiled on MOST 0808-471 turns it into a promising candidate to contribute to the GeV excess found by Pshirkov (2016). However, given the generally poor angular resolution of present $\gamma$-ray instruments, carrying multiwavelength observations is of paramount importance and should always be considered before jumping into conclusions about the nature of Fermi sources, in particular in the case of the field of WR 11 that is rich in nonthermal sources.

\subsection{Radio detected sources}

The areas surveyed to produce the catalog of sources listed as material accompanying this article correspond to the FoV circles (defined as the Half Power Beam Width) of the GMRT at the observed bands, centered at WR 11 with diameters of $3^{\circ}$ at $150 \mathrm{MHz}, 1.5^{\circ}$ at $325 \mathrm{MHz}$, and $0.75^{\circ}$ at $610 \mathrm{MHz}$. The detection threshold is given by the image rms (see Table 1).

The number of sources detected at 150,325 , and $610 \mathrm{MHz}$ resulted in 410, 224, and 66, respectively, yielding densities of 14,39 , and 41 sources - above $7 \mathrm{rms}$ - per square degree. The discrete sources at each band corresponded to 78, 82, and $69 \%$ of the sources fitted.

Of the 53 sources detected at least in two bands, 24 are detected at the three bands; 6 at only the 150 and $325 \mathrm{MHz}$ bands and 24 at only the 325 and $610 \mathrm{MHz}$ bands. Of this last group, four of these presented spectral index consistent with - or dominated by - thermal emission, whereas the rest showed spectral indices below -0.1 and are, therefore, most likely dominated by NT emission. In addition, 19 of these 53 sources are projected onto the Fermi excess region. The search for counterparts of those 19 sources resulted in correlations with stellar sources, like YSO (candidates), stars, and pre-main-sequence stars.

From a theoretical point of view, at low frequencies the NT emission (if existent) should be dominant, so we could expect that NT spectral indices should be very frequent, whereas spectral indices obtained at larger frequencies could be less negative due to "contamination" with thermal emission that becomes more relevant. Nonetheless, absorption/suppression effects might be important and these have a greater effect at low frequencies; the consequent decrease of the low-frequency emission implies that the spectral index obtained from the low-frequency range might be more positive than that obtained at higher frequencies. In fact, that tendency can be appreciated in Fig. 6.

Figure 6 (bottom) shows that almost all sources present a NT spectral index when estimated from the 325 and $610 \mathrm{MHz}$ fluxes, suggesting that any suppression/absorption effects that could be at work at those frequencies are not efficient or relevant. In contrast, Fig. 6 (top) shows that spectral indices estimated from the $325 \mathrm{MHz}$ fluxes and the $150 \mathrm{MHz}$ flux ULs also present a small number of sources with positive spectral indices. We interpret this as evidence that, at least for some sources, suppression/absorption effects could be more relevant at the 
lowest frequencies of this study. For the present study, the images were built to emphasize point sources, thus some part of the diffuse (possibly thermal) emission is not shown. The fact that the majority of the sources in Table 5 have NT spectral indices is probably due to an observational bias, as NT sources are stronger than thermal sources at low frequencies. Thus, the NT contribution prevails regardless of whether both contributions, thermal and NT, are present. Moreover, at lower frequencies the rms increases so less weak sources are revealed. The content of Table 5 results therefore from a combination of decreasing sensitivity threshold with frequency, and also angular resolution, and a weakening of thermal emission at lower frequencies with respect to the NT emission.

\section{Conclusions}

We studied the massive binary system WR 11 and its surroundings by means of dedicated radio interferometric observations from $150 \mathrm{MHz}$ to $1.4 \mathrm{GHz}$, plus archive data up to $230 \mathrm{GHz}$. Our data set, in particular the new GMRT data, constitute to date the most sensitive radio survey of this region, with unprecedented angular resolution.

Our analysis allowed us to draw the following inferences:

- From our GMRT observations we identified more than 400 radio emitters in the vicinity of WR 11, among which a significant fraction is detected in more than one band. A large percentage of these present a nonthermal spectrum, in agreement with the expectations at such low frequencies. We also show evidence suggesting that in many sources absorption/suppression processes significantly shape the SED at frequencies below $325 \mathrm{MHz}$.

- The radio spectrum of WR 11 confirms the predominance of thermal emission from $150 \mathrm{MHz}$ to $230 \mathrm{GHz}$. The results presented in this work constitute the only set of measurements leading to a spectral index determination down to $150 \mathrm{MHz}$ for a CWB to date. In this short-period binary system, the WCR is buried in the radio photospheres of the binary components, and nonthermal emission from it is expected to be suppressed by strong free-free absorption from the individual thermal winds. These results strongly suggest that the interpretation by Chapman et al. (1999) of a nonthermal component in the radio spectrum of WR 11 is misleading.

- The measured fluxes allowed us to derive a stellar mass-loss rate that is enough, according to the model by Reitberger et al. (2017), to explain a putative contribution of WR 11 to the Fermi excess.

- Our analysis allowed partial characterization of close-by source(s) at the position of MOST 0808-471 that presents intense, nonthermal radio emission, confirming its capability to participate in nonthermal processes. As a result, MOST 0808-471 deserves to be considered as a potential contributor to the $\gamma$-ray source identified by Pshirkov (2016). A detailed multiwavelength study however is needed to investigate its nature and potential capability to radiate at $\mathrm{GeV}$ energies.

Acknowledgements. We thank the staff of the GMRT that made these observations possible. GMRT is run by the National Centre for Radio Astrophysics of the Tata Institute of Fundamental Research. P.B. thanks Divya Overoi and Huib Intema for fruitful discussions on data reduction during her stay at the NCRA facility and Niruj Moham Ramanujam and Marcelo E. Colazo for help related with the pyBDSF tool handling. S.del.P. thanks the Stack Exchange community for the useful information available. This research has made use of the SIMBAD database, operated at CDS, Strasbourg, France and of NASA's Astrophysics Data System.

\section{References}

Ackermann, M., Ajello, M., Atwood, W. B., et al. 2015, ApJ, 810, 14

Anglada, G., Rodríguez, L. F., \& Carrasco-González, C. 2018, A\&ARv, 26, 3 Araudo, A. T., Romero, G. E., Bosch-Ramon, V., \& Paredes, J. M. 2007, A\&A, 476, 1289

Benaglia, P. 2016, Pub. Astron. Soc. Aust., 33, e017

Chapman, J. M., Leitherer, C., Koribalski, B., Bouter, R., \& Storey, M. 1999, ApJ, 518, 890

Crowther, P. A. 2007, ARA\&A, 45, 177

Cutri, R. M., Wright, E. L., Conrow, T., et al. 2012, VizieR Online Data Catalog: II $/ 311$

De Becker, M., \& Raucq, F. 2013, A\&A, 558, A28

De Becker, M., Benaglia, P., Romero, G. E., \& Peri, C. S. 2017, A\&A, 600, A47

De Becker, M., Isequilla, N. L., \& Benaglia, P. 2019, A\&A, 623, A163

Drew, J. E. 1990, in Properties of Hot Luminous Stars, ed. C. D. Garmany, ASP Conf. Ser., 7, 230

Eichler, D., \& Usov, V. 1993, ApJ, 402, 271

Gooch, R. 1996, in Astronomical Data Analysis Software and Systems V, eds. G. H. Jacoby \& J. Barnes, ASP Conf. Ser., 101, 80

Greisen, E. W. 2003, Astrophy. Space Sci. Lib., 285, 109

Hamaguchi, K., Corcoran, M. F., Pittard, J. M., et al. 2018, Nat. Astron., 2, 731

Intema, H. T. 2014, Astron. Soc. India Conf. Ser., 13, 469

Jones, P. A. 1985, MNRAS, 216, 613

Lamberts, A., Millour, F., Liermann, A., et al. 2017, MNRAS, 468, 2655

Leitherer, C., \& Robert, C. 1991, ApJ, 377, 629

Leitherer, C., Chapman, J. M., \& Koribalski, B. 1995, ApJ, 450, 289

Leitherer, C., Chapman, J. M., \& Koribalski, B. 1997, ApJ, 481, 898

Leser, E., Ohm, S., Füßling, M., et al. 2017, International Cosmic Ray Conference, 35,717

Martins, F., Schaerer, D., \& Hillier, D. J. 2005, A\&A, 436, 1049

McMullin, J. P., Waters, B., Schiebel, D., Young, W., \& Golap, K. 2007, in Astronomical Data Analysis Software and Systems XVI, eds. R. A. Shaw, F. Hill, \& D. J. Bell, ASP Conf. Ser., 376, 127

Montes, G., Pérez-Torres, M. A., Alberdi, A., \& González, R. F. 2009, ApJ, 705, 899

Montes, G., González, R. F., Cantó, J., Pé rez-Torres, M. A., \& Alberdi, A. 2011, A\&A, 531, A52

Morton, D. C., \& Wright, A. E. 1978, MNRAS, 182, 47P

Muijres, L. E., Vink, J. S., de Koter, A., Müller, P. E., \& Langer, N. 2012, A\&A, 537, A37

Murphy, T., Mauch, T., Green, A., et al. 2007, VizieR Online Data Catalog: VIII/82

Murphy, T., Sadler, E. M., Ekers, R. D., et al. 2009, VizieR Online Data Catalog: J/MNRAS/402/2403

North, J. R., Davis, J., Tuthill, P. G., Tango, W. J., \& Robertson, J. G. 2007, MNRAS, 380, 1276

Panagia, N., \& Felli, M. 1975, A\&A, 39, 1

Pittard, J. M. 2010, MNRAS, 403, 1633

Pittard, J. M., \& Dougherty, S. M. 2006, MNRAS, 372, 801

Pshirkov, M. S. 2016, MNRAS, 457, L99

Puls, J., Vink, J. S., \& Najarro, F. 2008, A\&ARv, 16, 209

Reimer, A., Pohl, M., \& Reimer, O. 2006, ApJ, 644, 1118

Reitberger, K., Reimer, A., Reimer, O., \& Takahashi, H. 2015, A\&A, 577, A100

Reitberger, K., Kissmann, R., Reimer, A., \& Reimer, O. 2017, ApJ, 847, 40

Reynolds, S. P., Gaensler, B. M., \& Bocchino, F. 2012, Space Sci. Rev., 166, 231

Rieger, F. M. 2017, AIP Conf. Ser., 1792, 020008

Rodríguez-Kamenetzky, A., Carrasco-González, C., Araudo, A., et al. 2016, ApJ, 818,27

Romero, G. E., Benaglia, P., \& Torres, D. F. 1999, A\&A, 348, 868

Romero, G. E., Torres, D. F., Kaufman Bernadó, M. M., \& Mirabel, I. F. 2003, A\&A, 410, L1

Runacres, M. C., \& Owocki, S. P. 2002, A\&A, 381, 1015

Rybicki, G. B., \& Lightman, A. P. 1979, Radiative Processes in Astrophysics (New York: John Wiley and Sons, Inc.)

Sault, R. J., Teuben, P. J., \& Wright, M. C. H. 1995, in Astronomical Data Analysis Software and Systems IV, eds. R. A. Shaw, H. E. Payne, \& J. J. E. Hayes, ASP Conf. Ser., 77, 433

Tadhunter, C. 2016, A\&ARv, 24, 10

Tavani, M., Sabatini, S., Pian, E., et al. 2009, ApJ, 698, L142

van der Hucht, K. A., Raassen, A. J. J., Mewe, R., et al. 2007, in Massive Stars in Interactive Binaries, eds. N. St. -Louis \& A. F. J. Moffat, ASP Conf. Ser., 367,159

White, R. L., \& Chen, W. 1995, in Wolf-Rayet Stars: Binaries; Colliding Winds; Evolution, eds. K. A. van der Hucht \& P. M. Williams, IAU Symp., 163, 438 Wright, A. E., \& Barlow, M. J. 1975, MNRAS, 170, 41 